\title{
Article
}

\section{Cohesion, team mental models, and collective efficacy: Towards an integrated framework of team dynamics in sport}

Filho, Edson, Tenenbaum, Gershon and Yang, Yanyun

Available at http://clok.uclan.ac.uk/14051/

Filho, Edson ORCID: 0000-0002-8548-4651, Tenenbaum, Gershon and Yang, Yanyun (2015) Cohesion, team mental models, and collective efficacy:

Towards an integrated framework of team dynamics in sport. Journal of Sports Sciences, 33 (6). pp. 641-653. ISSN 0264-0414

It is advisable to refer to the publisher's version if you intend to cite from the work. http://dx.doi.org/10.1080/02640414.2014.957714

For more information about UCLan's research in this area go to http://www.uclan.ac.uk/researchgroups/ and search for <name of research Group>.

For information about Research generally at UCLan please go to http://www.uclan.ac.uk/research/

All outputs in CLoK are protected by Intellectual Property Rights law, including Copyright law. Copyright, IPR and Moral Rights for the works on this site are retained by the individual authors and/or other copyright owners. Terms and conditions for use of this material are defined in the policies page. 


\section{Cohesion, team mental models, and collective efficacy: towards an integrated framework of team dynamics in sport}

Edson Filho ${ }^{\mathrm{ab}}$, Gershon Tenenbaum ${ }^{\mathrm{b}}$ \& Yanyun Yang ${ }^{\mathrm{b}}$

a Behavioral Imaging and Neural Dynamics Center, University of Chieti-Pescara, Chieti, Italy

${ }^{\mathrm{b}}$ Department of Educational Psychology and Learning Systems, Florida state University, Tallahassee, FL 32306, USA

Corresponding Author:

Edson Filho

Behavioral Imaging and Neural Dynamics Center

University of Chieti-Pescara

Via dei Vestini, 33 - 66013, Chieti, Italy

Email: soares_medeiros@yahoo.com.br

Phone: +39 333.304.5650 / +49 0176.3514.6564

Running title: Nomological network of team dynamics

Keywords: team dynamics, cohesion, team mental models, collective efficacy, nomological network

Number of figures: 2 
NOMOLOGICAL NETWORK OF TEAM DYNAMICS

Running head: NOMOLOGICAL NETWORK OF TEAM DYNAMICS

Cohesion, Team Mental Models, and Collective Efficacy: Towards an Integrated Framework of Team Dynamics in Sport 
NOMOLOGICAL NETWORK OF TEAM DYNAMICS

\begin{abstract}
A nomological network on team dynamics in sports consisting of a multi-framework perspective is introduced and tested. The aim was to explore the interrelationship among cohesion, team mental models (TMM), collective-efficacy (CE), and perceived performance potential (PPP). Three hundred and forty college-aged soccer players representing 17 different teams (8 female and 9 male) participated in the study. They responded to surveys on team cohesion, TMM, CE and PPP. Results are congruent with the theoretical conceptualization of a parsimonious view of team dynamics in sports. Specifically, cohesion was found to be an exogenous variable predicting both TMM and CE beliefs. TMM and CE were correlated and predicted PPP, which in turn accounted for 59\% of the variance of objective performance scores as measured by teams' season record. From a theoretical standpoint, findings resulted in a parsimonious view of team dynamics, which may represent an initial step towards clarifying the epistemological roots and nomological network of various team-level properties. From an applied standpoint, results suggest that team expertise starts with the establishment of team cohesion. Following the establishment of cohesiveness, teammates are able to advance teamrelated schemas and a collective sense of confidence. Limitations and key directions for future research are outlined.

Keywords: team dynamics, cohesion, team mental models, collective efficacy, nomological network.
\end{abstract}




\section{Cohesion, Team Mental Models, and Collective Efficacy: Towards an Integrated Framework of Team Dynamics in Sport}

Theoretical and empirical evidence supports the notion that cohesion, team mental models (TMM), and collective efficacy (CE) are positively associated with team performance (Fiore, Salas, Cuevas, \& Bowers, 2003; Mohammed, Ferzandi, \& Hamilton, 2010). However, scarce evidence exists on how these team level attributes are interrelated (Bandura, 1997; Ward \& Eccles, 2006). Specifically, a parsimonious nomological network involving these variables has not been tested yet (see Bandura 1997; Carron \& Hausenblas, 1998; Eccles \& Tenenbaum, 2007; Klimoski \& Mohammed, 1994; Mohammed et al., 2010; Salas, Sims, \& Burke, 2005). The present study revolves around this long standing research question, and aims at testing the notion of integrating main concepts of team dynamics into a multimodal yet parsimonious model. The aim was to propose and test an integrated view of team dynamics in sports. The organization framework for examining sport teams first proposed by Carron and Hausenblas (1998) served as an initial base to integrate cohesion, TMM, and CE under the same "meta-conceptual umbrella" (see Figure 1). Leading frameworks on cohesion (Carron, Widmeyer, \& Brawley, 1985), TMM (TMM; Eccles \& Tenenbaum, 2007), and CE (CE; Bandura, 1997) were considered in light of current evidence on team dynamics in sport psychology (Myers, Paiement, \& Feltz, 2007; Ward \& Eccles, 2006).

Team cohesion is a multidimensional phenomenon that includes both social and task components at an individual and team level of analysis (Carron et al., 1985). Social cohesion pertains to the notion of teammates bonding for social reasons, thus reflecting the extent that members of a team like to interact and enjoy each other's company. Task cohesion refers to the degree that members of a team bond to work together on a task, thus remaining united to achieve 
24 shared performance related goals. The notions of task and social cohesion are at the core of the

25 conceptual model of group cohesion proposed by Carron et al. (1985), which is an important part

26 of research on group dynamics in sport psychology (Carron \& Eys, 2012), and has been

27 incorporated in the nomological network of team dynamics proposed herein.

Of particular importance to this study is the notion that team cohesion is related to other

team-level constructs, such as TMM and CE (Eccles \& Tenenbaum, 2007; Fiore et al., 2003). In particular, we conceptualized team cohesion as an antecedent variable of team processes (e.g., TMM). To this extent, there is a general agreement that shared goals and a sense of social support and accountability antecedes the development of team related knowledge (Arrow, Poole, Henry, Wheelan, \& Moreland, 2004). This is also congruent with both theoretical reasoning and empirical findings suggesting that teammates’ social and task beliefs are essential to the development of TMM (Carron \& Hausenblas, 1998; Mathieu, Heffner, Goodwin, Salas, \& Cannon-Bowers, 2000).

TMM refer to the “collective task and team-relevant knowledge that team members bring to a situation” (Cooke et al., 2003, p. 153). TMM is thought to provide a heuristic route (i.e., rule of thumb) to members of a given team, thus accelerating teamwork coordination and optimizing team decision-making (Salas \& Klein, 2001). Accordingly, TMM is a multi-factorial phenomenon composed by declarative (i.e., “what to do”), procedural (i.e., "how to do”), and strategic information (i.e., macro-level knowledge; general game plan). Furthermore, teammates must possess and share both individual task-specific knowledge (i.e., idiosyncratic knowledge held by individual team members) and team-related knowledge (i.e., collective understanding of team procedures, strategies and contingency plans) in order to facilitate team coordination and performance (Filho, Gershgoren, Basevitch, Schinke, \& Tenenbaum, 2014; Klimoski \& 
47 Mohammed, 1994; Mohammed et al., 2010). Finally, TMM relies on coordinated division of

48 labor, which is primarily developed via implicit and explicit communication channels (Eccles \&

49 Tenenbaum, 2007; Lausic, Tenenbaum, Eccles, Jeong, \& Johnson, 2009).

50 TMM is at the core of the framework adapted from Carron and Hausenblas (1998), and

51 proposed herein. More specifically, TMM is conceptualized as being endogenous to cohesion

52 and exogenous to CE. To this extent, Bandura (1997) noted that CE is influenced by a myriad of

53 team level attributes, such as cohesion and team-related knowledge. This is also consistent with

54 the view that TMM is a process variable, which evolves over time and influences teammates' CE

55 beliefs (Eccles \& Tenenbaum, 2007; Eccles, 2010). Finally, this linkage is congruent with the

56 notion that teammates possessing more refined implicit and explicit coordination mechanisms

57 are more likely to evolve enduring efficacy beliefs (Mathieu et al., 2000; Peterson, Mitchell,

58 Thompson, \& Burr, 2000; Salas et al., 2005).

Defined as a “group’s shared belief in its conjoint capabilities to organize and execute the courses of action required to produce given levels of attainment” (Bandura 1997, p. 4), CE is

61 thought to be based on the same antecedents of self-efficacy, and is considered to mediate

62 between TMM and Perceived Performance Potential (PPP). To this extent, CE is theoretically 63 seen as a variable with predictive power over team performance (Bandura, 1997; Edmonds et al., 64 2009; Feltz, Short, \& Sullivan, 2008; Myers, Payment, \& Feltz, 2004). The notion of PPP, which 65 is correlated with objective performance scores as a reliability check, reflects a probabilistic 66 rather than deterministic view of performance in working groups in general, and in sport in 67 particular (Kamata, Tenenbaum, \& Hanin, 2002; Stumpf, Doh, \& Tymon, 2010). Foremost, this 68 notion is congruent with the self-reported measures utilized in the current study. 
The model proposed herein is based on Carron and Hausenblas (1998) organizational

70

71

72

73

74

75

76

77

78

79

80

81

82

83

84

85

86

87

88

89

90

91

framework of team dynamics in sports (see Figure 1). Nonetheless, certain aspects of the group

structure were not included in the model but indirectly measured through the consideration of member attributes (i.e., demographic factors, such as mean age, gender, players’ nationality) pertaining to the participants and their teams. Individual products were not considered here because the focus was at the team-level of analysis. Leadership and environmental factors, which have been associated with group dynamics in sport (Carron \& Eys, 2012), were also beyond the scope of the present study, which was centered on integrating cohesion, TMM and CE using structural equation modeling techniques. Accordingly, from a path-analytical perspective, this model postulates that (a) cohesion is an antecedent variable of TMM, and (b) TMM mediates the relationship between cohesion and CE, and (c) CE predicts PPP. In addition to being grounded in the seminal conceptualization of team dynamics in sports proposed by Carron and Hausenblas (1998), these directional paths are aligned with extant research suggesting that (a) team cohesion, TMM, and CE are intrinsically related constructs (Feltz et al., 2008; Mohammed et al., 2010), and (b) CE beliefs evolve once a sense of "team" has been established, and have a positive effect on performance (Bandura, 1997; Myers et al., 2004; Zaccaro et al., 1995).

From a factor analysis standpoint, the proposed model considers leading instruments designed to measure cohesion, TMM and CE. Also, we aimed for a parsimonious model with non-overlapping factors. Accordingly, we focused on measuring only the unique factorial contributions representing cohesion, TMM, and CE. In other words, potentially overlapping factors among the instruments utilized in this study were not considered. In particular, two subdimensions of TMM (i.e., General Task and Team Knowledge, Attitude Towards Teammate Task) as measured by the Team Assessment Diagnostic Measure (see Johnson et al., 2007) and 
92 one sub-dimension of CE (i.e., Team Unity) as measured by the Collective Efficacy

93 Questionnaire for Sports (see Short, Sullivan, \& Feltz, 2005), were not included in the model. To

94 this extent, a pilot study indicated statistical overlapping among these factors and cohesion

95 scores as measured by the Group Environment Questionnaire (see Carron et al., 1985).

96 Furthermore, peer-debriefing meetings among the authors led to a unanimous agreement

97 regarding the "conceptual equivalence” of the aforementioned factors. Hence, in the proposed 98 model cohesion portrays the idea of "team bonding," whereas TMM reflects the notion of

99 “coordination links” (i.e., synchronized action or effort among teammates during moments of 100 action) (see Eccles \& Tenenbaum, 2007). In essence, cohesion was conceptualized as having 101 social and task dimensions at both individual and group levels of analysis. TMM was thought to 102 reflect teammates’ (a) coordination links, (b) communication dynamics, and (c) resource sharing. 103 Finally, congruent with its theoretical roots, CE was thought to represent teammates’ perceived 104 “capability” of (a) ability, (b) effort, (c) persistence, and (d) preparation.

Altogether, our aim was to explore how various team properties are interrelated in a 106 factorial and structural fashion. Specifically, our aim was to propose and empirically test, 107 through structural equation modeling analyses, a nomological network of team dynamics in 108 sports as related to cohesion, TMM and CE. We also examined the intra and inter team 109 variability in cohesion, TMM, and CE scores of college soccer teams. This is in line with the 110 importance of properly examining nested data in social sciences in general, and in sport and 111 exercise psychology in particular (Feltz et al., 2008; Hershberger, 2006). Informing from the 112 reviewed literature, we hypothesized that: (a) the proposed model would adequately fit the data, 113 thereby supporting a parsimonious integrated view of team dynamics in sports, as related to 
114 cohesion, TMM and CE; and (b) path coefficients would vary by gender as men's and women's 115 group behaviors and beliefs tend to differ.

\section{Method}

\section{Participants}

An a priori power analysis was conceptualized to reflect the minimum number of cases

119 needed to propose and test a statistically valid model. Therefore, this analysis conducted for 120 testing model fit as a whole (i.e., $\Sigma=\Sigma(\theta)$; power $=.80, \alpha=.05$, RMSEA $=.00$ for null

121 hypothesis, and RMSEA = .05 for alternative hypothesis) defined the target sample size ( $\mathrm{n} \geq$ 122 214). Three hundred and forty college soccer players (178 females and 162 males) representing

12317 different teams affiliated with the National Association of Intercollegiate Athletics

124 participated in the study. The 17 teams were from 9 different states across the country and had a 125 mean of 20 athletes per team $(S D=3.48)$. Participants were 20.38 years old on average $(S D=$ 126 2.12), and had 14.66 years $(S D=3.92)$ of experience in soccer. On average, the participants had 127 been playing for their respective teams for 2.40 years $(S D=1.11)$. They had played a median of 12820 matches $(M=19.70, S D=1.39)$ over the season before taking part in the study. The majority 129 of participants were Caucasians (70.62\%), followed by “other races” (15.28\%), Black/Afro130 Americans (6.67\%), and Hispanic/Latinos (4.23\%).

\section{Instruments}

A demographic form was utilized to collect normative data. Additionally, the primary

133 choices of sport psychologists for studying cohesion (i.e., The Group Environment 134 Questionnaire) and CE (i.e., Collective Efficacy Questionnaire for Sports) were utilized. TMM 135 scores were assessed through the Team Assessment Diagnostic Measure (TADM) and PPP was 
measured through the Team Outcome Questionnaire (TOQ). Objective performance scores were obtained from the National Association of Intercollegiate Athletics’ official website.

Group Environment Questionnaire (GEQ; Carron et al., 1985). The Group

Environment Questionnaire is an 18-item measure, with anchors ranging from 1 (i.e., strongly disagree) to 9 (i.e., strongly agree) with higher scores reflecting greater perceptions of cohesion. Specifically, the Group Environment Questionnaire was designed to assess the degree of cohesion among team members in the following four dimensions: (a) Individual Attraction to the Group-Social (ATG-S, 5 items; e.g., "Some of my best friends are on this team.”), (b) Individual Attraction to the Group-Task (ATG-T, 4 items; e.g., "I like the style of play on this team.”), (c) Group Integration-Social (GI-S, 4 items; e.g., "Our team would like to spend time together in the off-season.”), and (d) Group Integration-Task (GI-T, 5 items; e.g., "Our team is united in trying to reach its performance goals.”). Carron, Brawley, and Widmeyer (1998) reported that Cronbach alphas for the four hypothetical dimensions of the Group Environment Questionnaire are for the most part satisfactory (i.e., $\alpha \geq .70$ ). They also reported extensive data suggesting the content, concurrent and predictive validities of the Group Environment Questionnaire. In this study, we used the original Group Environment Questionnaire by Carron et al. (1985), reversing the negatively worded items before computing the Cronbach alpha coefficient, which ranged from .56 to .75 . The entire scale’s alpha reliability was .85 .

Team Assessment Diagnostic Measure (TADM; Johnson et al., 2007). The Team Assessment Diagnostic Measure was designed to measure sharedness of team-related knowledge, thereby focusing on assessing similarity, rather than accuracy, of teammates perceived TMM. This 15-item questionnaire, with anchors ranging from 1 (i.e., strongly disagree) to 5 (i.e., strongly agree), was conceptualized to assess latent shared mental states 
159 (through its perceived functional roles) according to the following five factors: (a) General Task

160 and Team Knowledge (GTTK, 3 items; e.g., "My team knows specific strategies for completing various goals.”), (b) General Task and Communication Skills (GTC, 3 items; e.g., "My team consistently demonstrates effective listening skills.”), (c) Attitudes Toward Teammates and Task (GTT, 3 items; e.g., “My team takes pride in our work.”), (d) Team Dynamics and Interactions (TDI, 3 items; e.g., “My team solves problems that occur while doing our tasks.”), and (e) Team Resources and Working Environment (TRWE, 3 items; e.g., "My team knows the environmental constraints when we perform our tasks.”). These factors were found to have satisfactory reliability coefficients (i.e., $\alpha \geq .75$ ) and to account for $82 \%$ of the variance on sharedness of team-related knowledge (Johnson et al., 2007). Only General Task and Communication Skills, Team Dynamics and Interactions, and Team Resources and Working Environment were included in the proposed model. In this study, Cronbach alpha coefficients ranged from .77 to .84, and the entire scale’s alpha reliability was .91 .

Collective Efficacy Questionnaire for Sports (Collective Efficacy Questionnaire for Sports; Short et al., 2005). This instrument was designed to capture team member’s beliefs regarding their team capabilities in sport relevant tasks. Specifically, the Collective Efficacy Questionnaire for Sports is a 5-factor instrument containing 20 items measuring athletes’ confidence levels in their team’s (a) ability (4 items; e.g., “ability to outplay their opponents”), (b) effort (4 items; e.g., "to show a strong work ethic"), (c) preparation (4 items; e.g., "to devise a successful strategy”), (d) persistence (4 items; e.g., "to be persistent when obstacles are present”), and (e) unity capabilities (4 items; e.g., “to resolve conflicts”), on a Likert-type scale ranging from 1 (i.e., not at all confident) to 10 (i.e., extremely confident). “Unity” was not considered in the proposed model given that its items are similar to the ones measured by the 
182 Group Environment Questionnaire. Short et al. (2005) reported data demonstrating satisfactory

reliability, discriminant, convergent and predictive validity scores for the Collective Efficacy

Questionnaire for Sports. In the current study, Cronbach alpha coefficient ranged from .83 to .89, and the entire scale’s alpha reliability was .95.

Team Outcome Questionnaire (TOQ; Coleman, 2011). The Team Outcome

Questionnaire was utilized to assess perceived performance potential (PPP), which is a subjective account of a team's performance from the perspective of a team member. More specifically, PPP is a cross-domain topic pertaining to performance of working teams in business, sports, and the military (Stumpf et al., 2010). The Team Outcome Questionnaire consists of 9 items that describe goals related to team skills, strategy, effort, competitive outcomes, and fitness (e.g., "My team potential to accumulate its potential amount of victories.”). The Team Outcome Questionnaire uses a Likert-type scale ranging from 0 (i.e., low expectations) to 4 (i.e., high expectations) to measure PPP in team sports. Initially based on a content analysis of team performance expectations (see Brawley, Carron, \& Widmeyer 1992), the Team Outcome Questionnaire was found to be a unidimensional scale accounting for approximately 55\% of the variability on team performance expectation. In this study, Cronbach alpha coefficient was .89.

The notion of PPP was utilized in terms of coherence, given that all other constructs (i.e., cohesion, TMM and CE) were based on self-reported measures. In this regard, Chelladurai (2007) posited that subjective reports may better represent athletes’ performance experiences. Purely objective scores do not account for an outstanding performance from the opposing team, referee mistakes, among other situational and environmental factors (e.g., bad weather, home advantage, injury). 
Objective performance. All teams’ final year ranking and season record (i.e., average points per game as measured by the number of wins representing 3 points, ties representing 1 point, and losses representing 0 points) were obtained from the National Association of Intercollegiate Athletics official website and correlated with Team Outcome Questionnaire scores to assess the criterion-related validity of this instrument.

\section{Procedures}

Institutional Review Board approval was obtained prior to the commencement of this study. College soccer coaches, affiliated with the National Association of Intercollegiate Athletics, received an email detailing the objectives of the project. Telephone calls and personal contacts were posteriorly arranged aiming to build rapport with the coaches. A pool of 44 coaches (all representing teams in the regional and national finals), was initially contacted, with 17 agreeing to participate in the study. Upon permission from the coaches, a time was scheduled to meet their respective players. The players were informed about the overarching theme of the study and asked to sign the written informed consent. Following the completion of the consent form, participants received a package containing a copy of the Group Environment Questionnaire, Team Assessment Diagnostic Measure, Collective Efficacy Questionnaire for Sports, Team Outcome Questionnaire, and the demographic information form. Questionnaires were presented in a randomized order in an attempt to control for learning and motivational effects. Participants were instructed to complete each questionnaire individually, and to be honest and serious in their responses. They received an envelope to confidentially return their responses upon completion. The questionnaires were administered in a quiet environment (i.e., meeting rooms) to secure the comfort and privacy of the participants. Coaches did not remain in the room during data collection. Data were collected at the end of the season. Participants had 
228

229

230

231

232

233

234

235

236

237

238

239

240

241

242

243

244

245

246

247

248

249

250

played a median of 20 matches $(M=19.7, S D=1.39)$ over the season before taking part in the study. Moreover, data was deliberately collected one day before a decisive playoff game at the national tournament as teammates' beliefs assessed prior to competition have been found to reliably predict team performance (Myers, et al., 2007).

\section{Results}

\section{Demographic Analyses}

Demographic analyses indicated expressive nationality and ethnic diversity among the teams surveyed (i.e., 33.2\% international student-athletes). Starters were more likely to report higher levels of “attraction to group task” as measured by the Group Environment Questionnaire, and women's soccer teams showed a higher proportion of offensive players than male soccer teams ( $\left(28.2 \%\right.$ vs. $\left.{ }^{\dagger} 16.8 \% ; \chi 2=25.41 d f=3, p<.01\right)$.

\section{Psychometric Analyses}

Reliability Analyses. Descriptive statistics and estimates of internal consistency reliability are presented in Table 1 . Overall, means across items were above the $70^{\text {th }}$ percentile for each subscale. The reliability coefficient obtained for each scale was adequate (i.e., $\alpha \geq .85$ ). The alphas for the subscales ranged between .56 - .75 for the Group Environment Questionnaire, .77 - .84 for the Team Assessment Diagnostic Measure, and .83 - .89 for the Collective Efficacy Questionnaire for Sports. Item 17 (i.e., “Overcome distractions”) and 19 (i.e., “Devise a successful strategy”) were excluded from the Collective Efficacy Questionnaire for Sports questionnaire due to a small correlation with the other items represented in the "Effort" and “Preparation” subscales, respectively. Alpha coefficients for the Group Environment Questionnaire subscales were not ideal, particularly for the Attraction to Group Task (.56) and Attraction to Group Social (.63). These two subscales were excluded from the model proposed 
251 herein (see Figure 2). This is congruent with different interpretations of the Group Environment

252 Questionnaire, in which the instrument was found to assess two, and not four, latent factors

253 (Carless \& De Paola, 2000; Schutz et al., 1994). The maintenance of GI-T and GI-S in the model

254 proposed herein is (a) congruent with the overarching theoretical notion of social and task

255 cohesion, and (b) representative of participants' group views of cohesion.

256 Correlational Analyses. Correlation coefficients among the Team Outcome

257 Questionnaire and objective performance measures were positive and moderate- to-high, hence

258 supporting the predictive validity of this instrument. Specifically, Team Outcome Questionnaire

259 and team season record showed a $r=.77\left(R^{2}=.59\right)$, whereas Team Outcome Questionnaire and

260 the National Association of Intercollegiate Athletics's final ranking correlated positively with a $r$

$261=.55\left(R^{2}=.30\right)$. Correlation coefficients among Group Environment Questionnaire, Collective

262 Efficacy Questionnaire for Sports, Team Assessment Diagnostic Measure, and Team Outcome

263 Questionnaire composite scores ranged from .51 to .71. Overall, correlation coefficients were

264 higher among the subscales of each instrument, but relatively lower between subscales

265 measuring different constructs. Specifically, the correlations ranged from .35 - .62 for the Group

266 Environment Questionnaire and Team Assessment Diagnostic Measure, .29 - .62 for the Group

267 Environment Questionnaire and Collective Efficacy Questionnaire for Sports, and .36 - .66 for

268 the Team Assessment Diagnostic Measure and Collective Efficacy Questionnaire for Sports.

269 Correlations for the Team Outcome Questionnaire ranged from .31 - .54 with the Group

270 Environment Questionnaire, .53 - .56 with the Team Assessment Diagnostic Measure, and .53 -

271.61 with the Collective Efficacy Questionnaire for Sports (see Table 2). Altogether, these

272 findings support the notion that cohesion, TMM, CE and PPP are interrelated but not identical

273 constructs, thereby warranting the examination of the nomological network proposed herein. 
Multi-Level Assessment. Intraclass correlation coefficients were computed for each

275 subscale included in further analyses. Table 1 shows intraclass correlation coefficients for each

276 variable, ranging from .10 (for Persistence) to .32 (for GI-S) with the majority of the values

277 lower than .20. Collectively, these results warranted the adoption of multi-level analysis (see

278 Hershberger, 2006). We thus applied multi-level structural equation modeling techniques to the

279 sample data following the stepwise procedure recommended by Stapleton (2006). The analyses

280 were conducted using Mplus 7. Stapleton (2006) suggested that the multi-level structural

281 equation modeling should start with the model labeled as maximal model, which consists of two

282 levels (i.e., between and within levels). At both levels, all pairs of variables are correlated with

283 each other, as the purpose of this model is to decompose the observed covariance matrix into two

284 components: (a) the covariance matrix for the between level, and (b) the covariance matrix for

285 the within level. The maximal model did not converge to solutions, as the between level

286 covariance matrix was not found to be positively definite. In addition, most of the estimated

287 covariances among the variables at the between level were not statistically significant at alpha

288 level of .05. This is likely due to the small sample size for the between level (i.e., 17 teams) and

289 due to the homogeneity of the sampled teams (i.e., all teams participated in the national playoff

290 finals). Specifically, when the sample size for the between level is small $(<100)$ and

291 homogenous, the model tends to encounter convergence problems and the standard errors of the

292 between level parameters tend to be equally small (Maas \& Hox, 2005). Given that the maximal

293 model did not converge to proper solutions, we were unable to continue with the multi-level

294 SEM analysis. Instead, further data modelling were conducted based on single-level analysis. 


\section{Structural Equation Modeling}

A two-step approach was utilized to test the fit of the hypothesized full structural model (see Kline, 2011). The first step consisted of evaluating the measurement model. The second step consisted of evaluating and revising the structural model. Although continuous data were utilized in this study (i.e., the average of scores across the set of items composing the Group Environment Questionnaire, Team Assessment Diagnostic Measure, Collective Efficacy Questionnaire for Sports, and Team Outcome Questionnaire subscales), visual inspection of Q-Q plots suggested the data were not normally distributed. Multivariate kurtosis was 17.84 with $p<$ .01. We thus applied robust maximum likelihood estimation method for SEM analysis using Satorra-Bentler (S-B) correction for non-normality (Kline, 2011). In all tested models, the error variance of PPP was fixed as zero because this construct has only one indicator. Chi-square with S-B correction ( $\chi_{S-B}^{2}$ with non-significance indicating good fit), comparative fit index (CFI $\geq .95$ indicating good fit), root mean square error approximation (RMSEA $\leq .08$ indicating good fit), standardized root mean square residual (SRMR $\leq .06$ indicating good fit), and weighted root mean square residual (WRMR $\leq 1$ indicating good fit) were used to evaluate model-fit (Kline, 2011).

Measurement model. The measurement model associated with the hypothesized full structural model is presented in Figure 2. The tested model allowed for 30 degrees of freedom, with $\chi^{2}(30)=55.14, p<.01$, S-B correction factor of 1.21, CFI $=.986$, RMSEA $=.050$, SRMR $=$ .026 , and $W R M R=.505$. Except $\chi 2$, which is influenced by sample size, these results suggested reasonable model-data fit. Standardized factor loadings were significant and moderate-to-high ranging from .67 to .91 . Modification indices did not suggest any theoretical or statistically meaningful adjustments. Hence, this model was considered the final measurement model. 
Hypothesized structural model. The tested model (Structural Model 1) allowed for 33 degrees of freedom with $\chi^{2}(33)=122.83, p<.01$, a S-B correction factor of $1.24, C F I=.950$, $R M S E A=.089, S R M R=.063$, and $W R M R=1.292$ (see Table 3). This model did not fit adequately to the data. Modification indices and theoretical meaning were considered in proposing the revised structural model. In particular, two structural changes, one at a time, were added to the revised structural models. First, a direct effect from cohesion to CE was added. This is congruent with empirical and theoretical evidence suggesting that cohesion scores predict CE beliefs in team sports (Bandura 1997; Heuzé, Sarrazin, Masiero, Raimbault, \& Thomas, 2006). Second, a direct link between TMM and PPP was also added in an attempt to improve overall model fit. This modification is congruent with empirical findings regarding the overall positive impact of TMM on team outcomes (Mathieu et al., 2000; Mohammed et al., 2010).

Revised structural models. This revised structural model with a direct effect from cohesion to CE (Structural Model 2) allowed for 32 degrees of freedom, with $\chi^{2}(32)=71.75, p<$ .01 , a S-B correction factor of $1.21, C F I=.978, R M S E A=.060, S R M R=.033$, and $W R M R=$ .611. The revised structural model with both the direct effect from cohesion to CE and the direct effect from TMM to PPP (Structural Model 3) had 31 degrees of freedom, with $\chi^{2}(31)=55.79, p$ $<.01$, a S-B correction factor of $1.20, C F I=.986, R M S E A=.048, S R M R=.026$, and $W R M R=$ .502. Both models demonstrated adequate fit. A $\chi^{2}$ difference $\left(\Delta \chi^{2}\right)$ test was conducted to evaluate their relative fit. The $\Delta \chi^{2}(1)=13.07, p<.01$, suggesting that Model 3 fit significantly better than Model 2. Furthermore, a $\chi^{2}$ difference test was performed between Structural Model 3 and the measurement model with $\Delta \chi^{2}(1)=0.25, p>.05$. This result indicated that Structural Model 3 did not demonstrate a significantly worse fit to the data when compared to the measurement model, and that its structural component fit the data well. Standardized factor 
341 loadings were moderate-to-high and ranged from .68 to .90. The standardized coefficients

342 connecting factors were also moderate-to-high and ranged from .27 to .76. Modification indices

343 did not suggest any statistically meaningful adjustments. Given that this model represented a

344 plausible nomological network of team sports, the next step consisted of testing for alternative

345 statistical models. This is congruent with the importance of considering alternative explanations

346 for the data set, particularly in cross-sectional study designs (Hershberger, 2006).

$347 \quad$ Alternative Models. Alternative models are models with different specifications but

348 yielding similar fit (Hershberger, 2006). Such models provide alternatively meaningful

349 explanations for the inter-correlation among the latent factors considered in this study. Numerous

350 exploratory analyses of other theoretically plausible models, such as testing a correlational link

351 between CE-PPP (i.e., reciprocal determinism; Bandura, 1997) or reversing the directional path

352 (e.g., CE-TMM-CO), were conducted. However, no statistically reliable results were obtained.

353 We thus tested an equivalent alternative model to the Structural Model 3 by replacing the direct

354 effect from TMM to CE with the correlation between their disturbances. Accordingly, TMM and

355 CE were hypothesized as sharing covariance rather than representing a sequential process. This

356 alternative Model (Structural Model 4) yielded the same fit and factor loadings as Structural

357 Model 3, with 31 degrees of freedom, $\chi^{2}(31)=55.79, p<.01$, a S-B correction factor of 1.20 ,

$358 C F I=.986, R M S E A=.048, S R M R=.026$, and $W R M R=.502$. Noteworthy, we opted for

359 Structural Model 4 as the final solution. This model is in agreement with the overarching notion

360 that team-level properties tend to be functionally co-dependent, thus mutually influencing each

361 other (Bandura, 1997; Klimoski \& Mohammed, 1994). In effect, there is theoretical and

362 empirical evidence suggesting that more confident group units are more likely to possess

363 elaborate information sharing systems and vice-versa (Bandura, 1997; Little \& Madigan, 1997). 
Accordingly, Structural Model 4 was considered final (see Figure 2), hence supporting the concept of a parsimonious nomological network of team dynamics in sports. In particular, this model is grounded in the notion that (a) cohesion predicts TMM coordination links and CE efficacy beliefs, and (b) TMM and CE are correlated, mediate the CO-PPP relationship, and have a direct impact of moderate magnitude on PPP. Total variance accounted for TMM, CE and PPP was $58 \%$, $78 \%$, and $47 \%$, respectively.

\section{Multiple-Sample Analyses}

Measurement models by gender. A multiple-sample SEM was employed to test for gender invariance based on the Structural Model 4. Idiosyncratic models by gender yielded different but reasonable fit indices (Table 3). In particular, the measurement model for both females and males allowed for 30 degrees of freedom. For the female group, $\chi^{2}(30)=50.40, p=$ .01 , a S-B correction factor of $1.17, C F I=.980, R M S E A=.062, S R M R=.033$, and $W R M R=$ .545. For the male group, $\chi^{2}(30)=42.66, p=.06$, a S-B correction factor of $1.20, C F I=.985$, $R M S E A=.051, S R M R=.030$, and $W R M R=.375$. Given that both models demonstrated reasonable fit, additional constrained models were considered to test for measurement and structural invariance across genders.

Unconstrained measurement model. In the first step of the multiple-sample analysis an unconstrained model was examined. This model allowed for 60 degrees of freedom, with $\chi^{2}$ (60) $=92.94, p<.01$, a S-B correction factor of $1.18, C F I=.982, R M S E A=.057$, and $S R M R=.032$, and $W R M R=.472$. Taken together, these fit indices indicated adequate fit. Thus, the constrained measurement model was analyzed in the next step.

Constrained measurement model. The second step of the analysis involved a constrained model in which the factor loadings were equalized across groups. This model 
387 demonstrated reasonable fit, with $\chi^{2}(66)=95.01, p=.01$, a S-B correction factor of $1.20, C F I=$ $388.984, R M S E A=.051, S R M R=.036$, and $W R M R=.524$. A $\chi^{2}$ difference test revealed a nonsignificant increase in chi-square when compared to the unconstrained measurement model, $\Delta \chi^{2}$ (6) $=3.10, p>.05$. Accordingly, there was evidence of metric invariance (i.e., factor loadings invariance) across genders. Next, the tenability of equal structural coefficients (i.e., coefficients among factors) across groups was tested.

Unconstrained structural model. The measurement component of the unconstrained structural model was the same as that in the constrained measurement model. The path coefficients connecting factors were freely estimated for both groups. This model demonstrated adequate fit with $\chi^{2}(68)=95.91, p=.01$, a S-B correction factor of $1.19, C F I=.985, R M S E A=$ $.049, S R M R=.036$, and $W R M R=.518$. This model did not fit significantly worse than the constrained measurement model with $\Delta \chi^{2}(2)=.14, p>.05$. Thus, a constrained structural model to test for the equality of structural coefficients was analyzed in the next step.

Constrained structural model. This model was the same as the unconstrained structural model except that the five path coefficients connecting factors were constrained to be equal across groups. This model also fit the data reasonably with $\chi^{2}(73)=105.26, p<.01$, a S-B correction factor of $1.20, C F I=.982, R M S E A=.051, S R M R=.056$, and $W R M R=.753$. A $\chi^{2}$ difference test revealed a non-significant change in chi-square when compared to the constrained measurement model, $\Delta \chi^{2}(7)=10.25, p>.05$. Likewise, this model did not fit significantly worse than the unconstrained structural model, $\chi^{2}(5)=9.11, p>.05$. Altogether therefore, there was evidence of measurement and structural invariance across genders. The parameter estimates for the constrained structural model are given in Figure 2. 


\section{Discussion}

A nomological network of team dynamics considering cohesion, TMM and CE was proposed and tested. Overall, findings support the factorial and conceptual validities of an integrated framework of team dynamics in sport. Results also revealed expressive nationality diversity among the soccer teams surveyed, thereby reinforcing the importance of studies addressing multiculturalism in team sports. Demographic analyses also revealed that starters reported a higher level of “attraction to group task” as measured by the Group Environment Questionnaire. Starters are probably clearer of their roles than non-starters as playing time offer opportunities to evolve task-related knowledge (Eccles \& Tenenbaum, 2007). The lack of effect of other demographic factors on cohesion, TMM, CE, and PPP scores may be linked to the homogeneity of the sampled population. The majority of the teams $(n=12)$ were in the top-16 in the country, and the remaining teams $(n=5)$ were region finalists.

The observation of moderate to high correlation coefficients among sub-factors of cohesion, TMM, and CE offered initial validation to the nomological network of team dynamics in sports proposed herein. The measurement model obtained is congruent with the organizational framework for examining sport teams offered by Carron and Hausenblas (1998). The final modified Structural Model 4 allowed adequate model fit by incorporating the notion that both TMM and CE have a direct impact on PPP. This final model (i.e., Structural Model 4) supports the notion of a parsimonious nomological network of team dynamics in sports, as related to cohesion, TMM and CE.

The theoretical view of team dynamics in sports presented herein is consistent with an extensive body of literature on the predictive power of task-shared knowledge and CE on performance measures (Fiore et al., 2003; Salas \& Klein, 2001; Bandura, 1997). Additionally, 
433 this final model reflects the notion that cohesion antecedes team processes (e.g., TMM, CE), 434 thereby lending support for Carron and Hausenblas’ (1998) conceptualization of team dynamics

435 in sports. Indeed, research has consistently shown that teammates' agreement on social and task436 related behaviors may antecede the development of team mental "schemas" and group-level 437 confidence (Mathieu et al., 2000). To this extent, Eccles and Tenenbaum (2007) posited that the 438 allocation of social and task responsibilities antecede the development of implicit and explicit 439 processes in sport teams. Empirical evidence is also in favor of the notion that cohesion scores 440 predict CE beliefs in team sports (Heuzé et al., 2006).

The final model illustrated in Figure 2 is also congruent with the notion that CE is 442 influenced by a myriad of other team-level attributes (Bandura 1997; Zacarro et al., 1995). In 443 particular, CE beliefs were found to be anteceded by cohesion scores and correlated with TMM 444 scores. In this regard, Bandura (1997) posited that cohesion is a major source of CE, which is 445 also associated with socio-cognitive variables, such as TMM. In this regard, Bandura (1997) 446 posited that cohesion is a major source of CE, which is also associated with socio-cognitive 447 variables, such as TMM. Hence, training sessions tailored to evolve team coordination and 448 communications links are likely to enhance a team's efficacy beliefs while also impacting team 449 performance.

450 Theoretically, the parsimonious view of team dynamics proposed herein may represent an 451 initial step towards clarifying the epistemological and nomological network roots of various 452 team-level properties. Theoretical models in sport sciences should focus on clarifying 453 (conceptually and statistically) the unique factorial contributions of its underlying latent factors. 454 For instance, the model proposed herein is statistically valid and supports the tested notion that 455 TMM is represented by coordination, communication, and team's resources networking. This 
may be seen as an initial step towards clarifying the unique antecedents of TMM - where the epistemological traits and anteceding variables are not yet clear (Cooke et al., 2003; Johnson et al., 2007). More specifically, different authors have proposed numerous conceptual frameworks describing hypothetical variables underlying the notion of TMM. Although conceptually appealing, these frameworks are primarily based on face-validity, thereby lacking statistical corroboration (Klimoski \& Mohammed, 1994; Mohamed et al., 2010; Salas et al., 2005). Future studies should therefore expand the analysis of TMM in an attempt to establish the unique variables anteceding this group level phenomenon.

From an applied standpoint, findings from this study illustrate the importance of (a) investing in the development of team cohesion in sports as this team attribute antecedes TMM and CE, and (b) TMM to team performance and confidence. Accordingly, results suggest that team expertise starts with the establishment of positive social relations (social cohesion), and task cohesion (i.e., teammates sharing the same task goals). Specifically, the large effect size found for the cohesion-TMM and cohesion-CE relationships illustrates the importance of performance enhancement activities aimed at improving team cohesiveness. Following the establishment of cohesiveness levels, teammates are able to advance team-related schemas and a collective sense of confidence. Hence, activities promoting heuristic (e.g., implicit and explicit) communication links, and a "team belief” on its capability to accomplish outcomes are subsequent steps in evolving team expertise.

Team cohesion representing the initial stage of the proposed conceptual framework reinforces the importance of preventing social isolation and attachment problems in team sports (Carron et al., 1985; Carron \& Eys, 2012). Low social cohesion may create negative affect and aggravate communication problems, thereby hindering the development of TMM. Similarly, low 
479 task cohesion may decrease members’ contribution and perceived responsibility, thus resulting in 480 lack of effort and inefficient coordination mechanisms (Eccles, 2010). Organizational and 481 individual orientations aimed at preventing the development of "social cliques", along with the 482 establishment of challenge goals and group-level productive norms, are important in building 483 team cohesion (Carron \& Eys, 2012).

$484 \quad$ The notion that TMM and CE are positively related is consistent with research findings 485 on working groups' coordination links and efficacy beliefs (Mathieu et al., 2000; Peterson et al., 486 2000). For instance, Mathieu et al. (2000) found that communication breakdowns are less likely 487 to happen in highly confident military units. Within the sport context, Lausic et al. (2009) 488 observed that more successful teams possess more homogenous models of communicating 489 emotional and action verbal and non-verbal messages. Hence, performance enhancement 490 consultants should target vicarious and verbal persuasion techniques (e.g., video-analysis, 491 motivational lectures) aiming at concomitantly addressing teammates’ confidence beliefs and 492 verbal and non-verbal communication skills. Men’s and women's soccer teams differed in their distribution of players by position. In 494 particular, women's teams showed a higher proportion of offensive players than male teams. 495 These differences warranted adoption of multiple-sample SEM procedures aimed at testing for 496 gender invariance given that in team sports each position has different objectives and demands 497 (Filho et al., 2014). Although presents results revealed measurement and structural invariance 498 across genders, a further study addressing a more heterogeneous sample may reveal gender 499 effects on team-level properties. Indeed, the analysis performed herein targeted the covariance 500 structure only (i.e., loadings, path coefficients). Accordingly, it is plausible that males and 501 females have a different means on the latent variables. Again, the athletes' surveyed represented 
502 the top performers in their conference, thereby a ceiling-effect on athletes' mental skills may

503 have "masked" a gender effect on the nomological network proposed herein. Accordingly, it is

504 likely that a future study may reveal a different interrelationship among cohesion-TMM-CE-PPP.

505 For instance, CE may have a larger impact on PPP for women’s soccer teams, whereas TMM

506 may be better predictor of performance for men’s soccer teams. In this regard, research has

507 shown that males and females differ in their emphasis on task oriented behavior, as well as on

508 their cohesiveness and collective efficacy dynamics (Chelladurai, 2007; Feltz et al., 2008; Schutz

509 et al., 1994).

510 Caution is warranted in generalizing these findings to other interactive sports,

511 competition levels, and different periods within a competitive season. Another limitation pertains

512 to the non-inclusion of the interrelationship between coaches' leadership behaviors and team

513 cohesion in sports. Coaching leadership is a vast topic and has been extensively studied

514 elsewhere (see Martens, 2004). Furthermore, the proposed model should be considered in terms

515 of its theoretical roots (i.e., socio-cognition). For instance, models grounded in dynamic systems

516 perspectives (e.g., eco-dynamical, course of action frameworks) may also represent valid

517 interpretations of team dynamics. The adoption of the expert-novice paradigm may expose

518 differences among “top” and "bottom” teams while also allowing the implementation of

519 multilevel models. Again, our dataset was homogeneous in nature and ultimately reflected our

520 target sample (i.e., top ranked teams). The reliance on modification indices moved the analysis

521 from a confirmatory to (at least) partially exploratory standpoint. Therefore, other models may

522 be plausible and longitudinal studies in particular, rather than cross-sectional, may offer

523 alternative views on how cohesion, TMM and CE are inter-related and exogenous or endogenous

524 to each other. Specifically, in the present cross-sectional study, all variables were measured (at 
525 the same time) at the end of the season, thus preventing the assessment of cyclical relationships

526 (involving cohesion, TMM, CE, and PPP) likely to change over time. Despite these limitations,

527 this study addressed a historically and scientifically pondered question of many leading scholars

528 in the field of group dynamics. In fact, this study is aligned with the need for theory integration

529 within the psychological domain (Gigerenzer, 2010). On this note, Waltkins (1984, p. 86)

530 observed that "psychologists treat other people’s theories like toothbrushes - no self-respecting

531 person wants to use anyone else's”. Accordingly, the nomological network of team sports

532 proposed herein may represent an initial step towards clarifying the epistemological and

533 nomological network roots of various team-level properties. Finally, findings from this study

534 also provide applied guidelines to evaluate and improve performance of highly interactive and

535 complex team units.

536

Perhaps more importantly, this study leads to further questions on "how multiple minds

537 work in synchrony” towards excellence and conflict resolution. Targeting different sub-

538 population groups (e.g., competition levels, cross/multi-cultural studies) and conceptual roots

539 (e.g., dynamic systems) may allow further revisions of parsimonious integrated models of team

540 dynamics in sport psychology. Addressing different working groups (e.g., military units, medical

541 teams) and considering models proposed in the I/O psychology may evolve a nomothetic, cross-

542 domain view of team dynamics. Implementation of longitudinal quantitative approaches (e.g.,

543 longitudinal growth models) may reveal how team dynamics change over time, particularly in

544 regards to the nomological network pertaining to cohesion, TMM and CE. For instance,

545 addressing how performance (i.e., output) re-inform teammates’ appraisals (i.e., new inputs) on

546 their cohesiveness, TMM, and CE beliefs may reveal how circular loops of influence

547 continuously reshape team dynamics. Consideration of newly developed instruments for 
548 cohesion (Eys, Carron, Bray, \& Brawley, 2007) and TMM (see Gershgoren, 2012) may

549 strengthen the validity of a statistically parsimonious view of team dynamics in sports. Testing

550 for the specific effects pertaining to the sub-factors of cohesion (i.e., task and social), TMM and

551 CE are also important steps for future research.

552 


\section{References}

Arrow, H., Poole, M. S., Henry, K. B., Wheelan, S., \& Moreland, R. (2004). Time, change, and development: The temporal perspective on groups. Small Group Research, 35, 73-105. doi:10.1177/1046496403259757

Bandura, A. (1997). Self-efficacy: The exercise of control. New York, NY: W.H. Freeman.

Brawley, L. R., Carron, A. V., \& Widmeyer, W. N. (1992). The nature of group goals in sport teams: A phenomenological analysis. The Sport Psychologist, 6, 323-333.

Carless, S. A., \& De Paola, C. (2000). The measurement of cohesion in work teams. Small Group Research, 31, 71-88. doi:10.1177/104649640003100104

Cannon-Bowers, J., \& Bowers, C. (2006). Applying work team results to sports teams: Opportunities and cautions. International Journal of Sport and Exercise Psychology, 4, 363-369.

Carron, A. V., Brawley, L. R., \& Widmeyer, W. N. (1998). Measurement of cohesion in sport and exercise. In J. L. Duda (Ed.). Advances in sport and exercise psychology measurement (pp. 213-226). Morgantown, WV: Fitness Information Technology.

Carron, A. V., \& Eys, M. A. (2012). Group dynamics in sport. (4th ed.), Morgantown, WV: Fitness Information Technology.

Carron, A. V., \& Hausenblas, H. A. (1998) Group dynamics in sport (2nd ed.). Morgantown, WV: Fitness Information Technology.

Carron, A. V., Widmeyer, W. N., \& Brawley, L. R. (1985). The development of an instrument to assess cohesion in sport teams: The group environment questionnaire. Journal of Sport Psychology, 7, 244-266. 
Chelladurai, P. (2007). Leadership in Sports. In G. Tenenbaum \& R. C. Eklund (Eds.), Handbook of sport psychology (3rd ed., pp. 113-135). New Jersey: John Wiley \& Sons.

Coleman, J. (2011). A functional model of team leadership for sport. The Florida State University). ProQuest Dissertations and Theses, 136.

Cooke, N. J., Kiekel, P. A., Salas, E., Stout, R., Bowers, C., \& Cannon-Bowers, J. (2003). Measuring team knowledge: A window to the cognitive underpinnings of team performance. Group Dynamics: Theory, Research, and Practice, 7, 179-199. doi: 10.1037/1089-2699.7.3.179

Eccles, D. (2010). The coordination of labour in sports teams. International Review of Sport and Exercise Psychology, 3, 154-170. doi:10.1080/1750984X.2010.519400

Eccles, D. W., \& Tenenbaum, G. (2007). A social-cognitive perspective on team functioning in sport. In G. Tenenbaum \& R. C. Eklund (Eds.), Handbook of sport psychology (3rd ed., pp. 264-283). Hoboken, NJ: John Wiley \& Sons, Inc.

Edmonds, W. A., Tenenbaum, G., Kamata, A., \& Johnson, M. B. (2009). The role of collective efficacy in adventure racing teams. Small Group Research, 40, 163-180. doi:10.1177/1046496408328489

Eys, M. A., Carron, A. V., Bray, S. R., \& Brawley, L. R. (2007). Item wording and internal consistency of a measure of cohesion: The group environment questionnaire. Journal of Sport \& Exercise Psychology, 29, 395-402.

Feltz, D. L., Short, S. E., \& Sullivan, P. J. (2008). Self-efficacy in sport. Champaign, IL, US: Human Kinetics. 
605

606 607

608

609

610

611

612

613

614

615

616

617

618 619

Fiore, S. M., Salas, E., Cuevas, H. M., \& Bowers, C. A. (2003). Distributed coordination space: Toward a theory of distributed team process and performance. Theoretical Issues in Ergonomics Science, 4, 340-364. doi:10.1080/1463922021000049971

Filho, E., Gershgoren, L., Basevitch, I., Schinke, R., \& Tenenbaum, G. (2014). Peer Leadership and Shared Mental Models in a College Volleyball Team: A Season Long Case Study. Journal of Clinical Sport Psychology, 8, 184-203.

Gershgoren, L. (2012). The development and validation of the Shared Mental Models in Team Sports Questionnaire. (The Florida State University). ProQuest Dissertations and Theses, 320.

Gigerenzer, G. (2010). Personal reflections on theory and psychology. Theory \& Psychology, 20, 733-743. doi :http://dx.doi.org/10.1177/0959354310378184

Hershberger, S. L. (2006). The problem of equivalent structural models. In G. R. Hancock, \& R. O. Mueller (Eds.). Structural equation modeling: A second course (pp.13-41). Connecticut: Information Age Publishing.

Heuzé, J., Sarrazin, P., Masiero, M., Raimbault, N., \& Thomas, J. (2006). The relationships of perceived motivational climate to cohesion and collective efficacy in elite female teams. Journal of Applied Sport Psychology, 18, 201-218. doi:10.1080/10413200600830273

Johnson, T. E., Lee, Y., Lee, M., OConnor, D. L., Khalil, M. K., \& Huang, X. (2007). Measuring sharedness of team-related knowledge: Design and validation of a shared mental model instrument. Human Resource Development International, 10, 437-454. doi:10.1080/13678860701723802

Kamata, A., Tenenbaum, G., \& Hanin, Y. L. (2002). Individual zone of optimal functioning (IZOF): A probabilistic estimation. Journal of Sport \& Exercise Psychology, 24, 189-208. 
620

621

622

623

624

625

626

627

628

629

630

631

632

633

634

635

636

637

638

639

640

641

Klimoski, R., \& Mohammed, S. (1994). Team mental model: Construct or metaphor? Journal of Management, 20, 403-437. doi:10.1016/0149-2063(94)90021-3

Kline, R. B. (2011). Principles and practice of structural equation modeling (3rd ed.). New York, NY: The Guilford Press.

Lausic, D., Tennebaum, G., Eccles, D., Jeong, A., \& Johnson, T. (2009). Intrateam Communication and Performance in Doubles Tennis. Research Quarterly for Exercise and Sport, 80, 281-290.

Lirgg, C. D. (1991). Gender differences in self-confidence in physical activity: A meta-analysis of recent studies. Journal of Sport \& Exercise Psychology, 13, 294-310. Retrieved from http://search.proquest.com/docview/618084666?accountid=4840

Little, B. L., \& Madigan, R. M. (1997). The relationship between collective efficacy and performance in manufacturing work teams. Small Group Research, 28, 517-534. doi:10.1177/1046496497284003

Martens, R. (2004). Successful coaching. (3rd ed.). Champaign, IL: Human Kinetics.

Maas, C. J. M., \& Hox, J. J. (2005). Sufficient sample sizes for multilevel modeling. Methodology, 1, 86-92.

Mathieu, J. E., Heffner, T. S., Goodwin, G. F., Salas, E., \& Cannon-Bowers, J. (2000). The influence of shared mental models on team process and performance. Journal of Applied Psychology, 85, 273-283. doi:10.1037/0021-9010.85.2.273

Mohammed, S., Ferzandi, L., \& Hamilton, K. (2010). Metaphor no more: A 15-year review of the team mental model construct. Journal of Management, 36, 876-910. doi:10.1177/0149206309356804 
642 Myers, N. D., Payment, C. A., \& Feltz, D. L. (2004). Reciprocal relationships between collective

643

644

645

646

647

648

649

650

651

652

653

654

655

656

657

658

659

660

661

662

663

664

efficacy and team performance in womens ice hockey. Group Dynamics: Theory,

Research, and Practice, 8, 182-195. doi:10.1037/1089-2699.8.3.182

Myers, N.D., Paiement, C.A., \& Feltz, D.L. (2007). Regressing team performance on collective efficacy: Considerations of temporal proximity and concordance.

Measurement in Physical Education and Exercise Science, 11, 1-24.

Peterson, E., Mitchell, T. R., Thompson, L., \& Burr, R. (2000). Collective efficacy and aspects of shared mental models as predictors of performance over time in work groups. Group Processes \& Intergroup Relations, 3, 296-316. doi:10.1177/1368430200033005

Salas, E., \& Klein, G. (2001) Expertise and naturalistic decision making: An overview. In E. Salas \& G. Klein, (Eds.), Linking expertise and naturalistic decision making (pp. 3-8). Hillsdale, NJ: Lawrence Erlbaum.

Salas, E., Sims, D. E., \& Burke, C. S. (2005). Is there a "big five" in teamwork? Small Group Research, 36, 555-599. doi:10.1177/1046496405277134

Short, S. E., Sullivan, P., \& Feltz, D. L. (2005). Development and preliminary validation of the collective efficacy questionnaire for sports. Measurement in Physical Education and Exercise Science, 9, 181-202. doi:10.1207/s15327841mpee0903_3

Schutz, R. W., Eom, H. J., Smoll, F. L., \& Smith, R. E. (1994). Examination of the factorial validity of the group environment questionnaire. Research Quarterly For Exercise \& Sport, 65, 226-236.

Stapleton, L. M. (2006). Using multilevel structural equation modeling techniques with complex sample data. In G. R. Hancock, \& R. O. Mueller (Eds.). Structural equation modeling: A second course (pp.345-383). Connecticut: Information Age Publishing. 
665 Stumpf, S. A., Doh, J. P., \& Tymon, W. (2010). The Strength of HR Practices in India and Their 666 Effects on Employee Career Success, Performance, and Potential. Human Resource

667 Management, 49, 353-375.

668 Tenenbaum, G., Eklund, R., \& Kamata, A. (2012). Measurement in sport and exercise 669 psychology. Human Kinetics, Champaign, IL.

670 Ward, P., \& Eccles, D. W. (2006). A commentary on "team cognition and expert teams:

671 Emerging insights into performance for exceptional teams." International Journal of

672 Sport and Exercise Psychology, 4, 463-483. doi:10.1080/1612197X.2006.9671808

673 Watkins, M. J. (1984). Models as toothbrushes. Behavioral \& Brain Sciences, 7, 86.

674 doi:10.1017/S0140525X00026303

675 Zaccaro, S. J., Blair, V., Peterson, C., \& Zazanis, M. (1995). Collective efficacy. In J. E. 676 Maddux (Ed.), Self-efficacy, adaptation, and adjustment: Theory, research, and application (pp. 305-328). New York: Plenum. 


\section{(A) Founding Conceptualization of Team Dynamics in Sports}

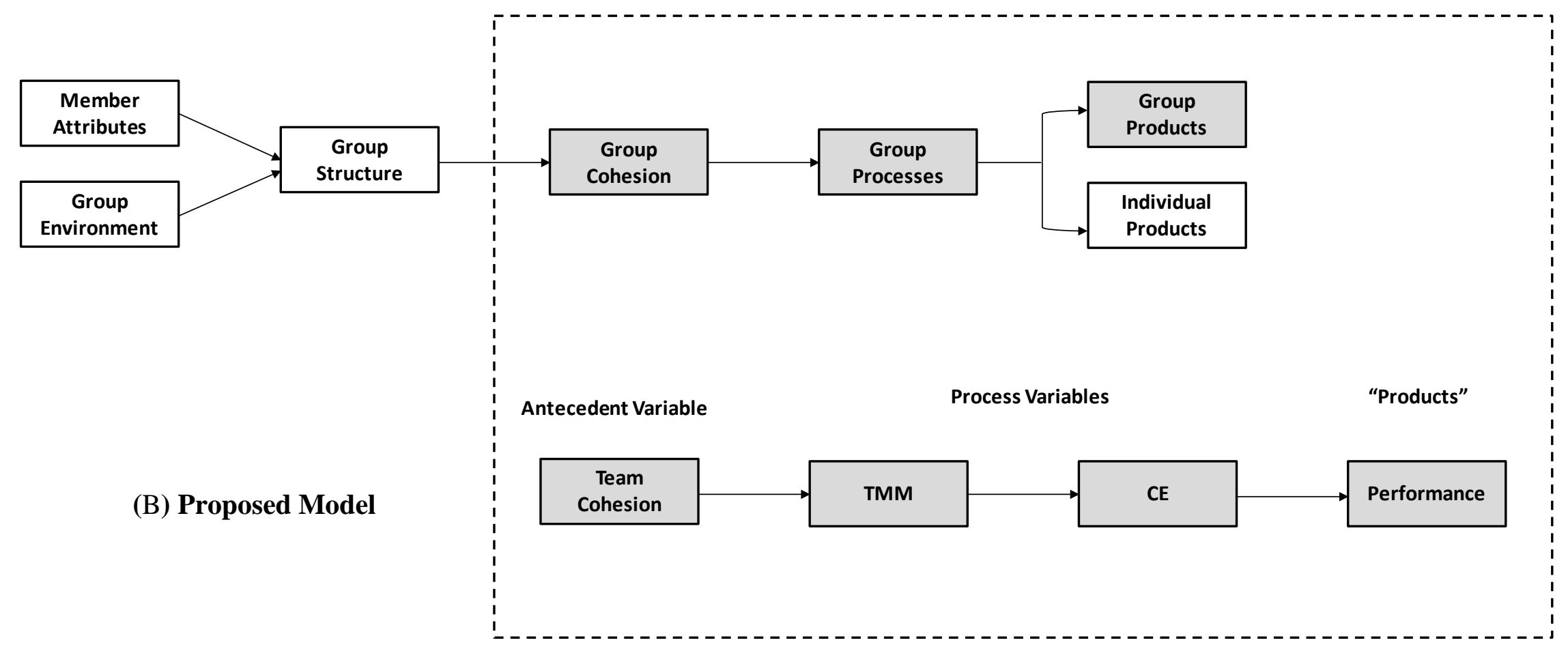

Figure 1. (A) “Conceptual Framework for Examining Sport Teams” by A. V. Carron and H. Hausenblas, 1998, Group dynamics in sport, p. 166. Copyright 1998 by Fitness Information Technology. Adapted with permission. (B) Proposed Nomological Network of Team Dynamics in Sports.

Note. Group structure was indirectly measured through the consideration of demographic information pertaining to the participants and their teams. Individual products were not considered here because the focus was at the team-level of analysis. 

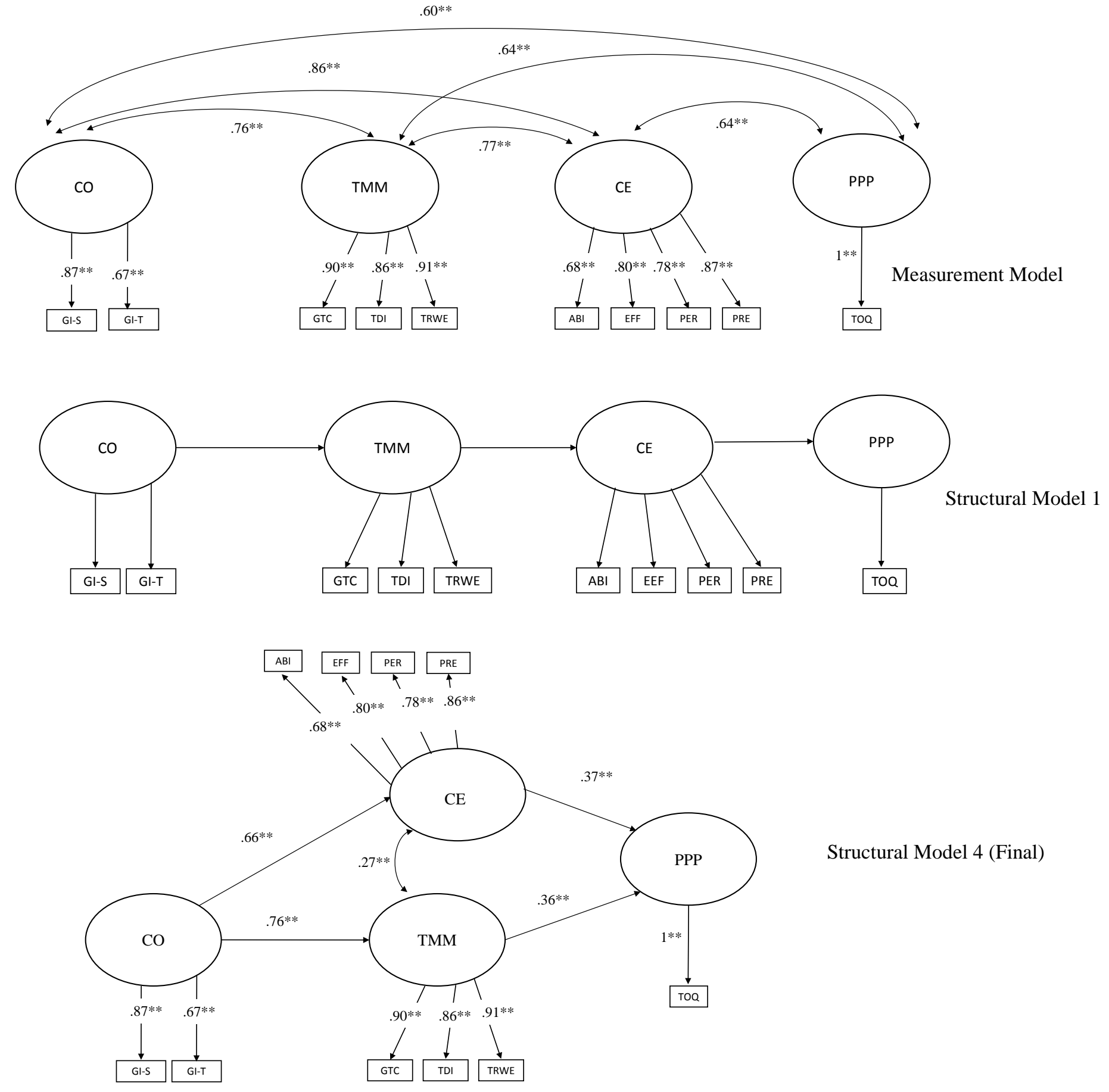

Structural Model 4 (Final)

$* * p<.01$

Figure 2. Integrated Nomological Network of Team Dynamics in Sport

Note.: Cohesion: Group Integration-Social (GI-S). Group Integration Task (GI-T). TMM: General Task and Communication (GTC). Team Dynamics Interactions (TDI). Team Resources and Working Environment (TRWE). CE: Ability (ABI). Effort (EFF). Persistence (PER). Preparation (PRE). Performance Expectation (PPP). 
Table 1

Descriptive Statistics and Reliability Estimates and Intra-Class Correlation Coefficients (ICC) for the GEQ, TADM, CEQS and TOQ

\begin{tabular}{|c|c|c|c|c|c|}
\hline \multirow[b]{2}{*}{ Scale } & \multicolumn{4}{|c|}{ Descriptive Statistics } & \multirow[b]{2}{*}{$I C C$} \\
\hline & $M$ & $S D$ & Range & Alpha & \\
\hline \multicolumn{6}{|l|}{ GEQ } \\
\hline ATG-T & 6.96 & 1.59 & $2-9$ & .56 & .14 \\
\hline ATG-S ${ }^{b}$ & 7.29 & 1.45 & $1-9$ & .63 & .12 \\
\hline GI-T & 6.79 & 1.44 & $2-9$ & .75 & .15 \\
\hline GI-S ${ }^{d}$ & 6.70 & 1.65 & $1-9$ & .72 & .32 \\
\hline Total GEQ & 6.94 & 1.19 & $3-9$ & .85 & .22 \\
\hline \multicolumn{6}{|l|}{ TADM } \\
\hline $\mathrm{GTC}^{\mathrm{e}}$ & 3.84 & .68 & $2-5$ & .84 & .13 \\
\hline $\mathrm{TDI}^{\mathrm{f}}$ & 3.89 & .65 & $2-5$ & .81 & .13 \\
\hline TRWE $^{\mathrm{g}}$ & 3.99 & .64 & $1-5$ & .77 & .10 \\
\hline Total TADM & 3.91 & .59 & $2-5$ & .88 & .16 \\
\hline \multicolumn{6}{|l|}{ CEQS } \\
\hline Ability & 8.30 & 1.36 & $3-10$ & .89 & .10 \\
\hline Effort & 8.44 & 1.35 & $3-10$ & .83 & .12 \\
\hline Persistence & 8.27 & 1.41 & $2-10$ & .87 & .19 \\
\hline Preparation & 8.53 & 1.32 & $2-10$ & .83 & .13 \\
\hline Total CEQS & 8.33 & 1.20 & $3-10$ & .95 & .10 \\
\hline TOQ & 3.29 & .54 & $1-4$ & .89 & .24 \\
\hline
\end{tabular}

Note.: ${ }^{\mathrm{a}}$ Individual Attraction to the Group-Task. ${ }^{\mathrm{b}}$ Individual Attraction to the Group-Social. ${ }^{\mathrm{c}}$ Group Integration Task. ${ }^{\mathrm{d}}$ Group Integration-Social. ${ }^{\mathrm{e}}$ General Task and Communication. ${ }^{\mathrm{f}}$ Team Dynamics Interactions. ${ }^{\mathrm{g}}$ Team Resources and Working Environment. 
Table 2

Correlation Matrix among GEQ, CQES, TADM Subscale, and TOQ

\begin{tabular}{|c|c|c|c|c|c|c|c|c|c|c|c|}
\hline & ATG_T ATG_S & $\overline{\text { GI_T }}$ & $\overline{\text { GI_S }}$ & $\overline{\text { GTC }}$ & TDI & TRWE & Ability & Effort & Persistence & Preparation & $\overline{\text { TOQ }}$ \\
\hline$\overline{\text { ATG-T }^{\mathrm{a}}}$ & .47 & .44 & .24 & .35 & .38 & .41 & .35 & .44 & .40 & .43 & .35 \\
\hline ATG-S ${ }^{b}$ & & .52 & .49 & .39 & .46 & .44 & 29 & .42 & .36 & .38 & .31 \\
\hline GI-T ${ }^{c}$ & & & .58 & .59 & .62 & .62 & .44 & .62 & .55 & .56 & .54 \\
\hline GI-S ${ }^{d}$ & & & & .47 & .54 & .51 & .29 & .42 & .32 & .40 & .37 \\
\hline GTC $^{e}$ & & & & & .70 & .66 & .36 & .60 & .52 & .53 & .53 \\
\hline $\mathrm{TDI}^{\mathrm{f}}$ & & & & & & .76 & .42 & .60 & .55 & .55 & .53 \\
\hline TRWE $^{\mathrm{g}}$ & & & & & & & .45 & .57 & .54 & .55 & .56 \\
\hline Ability & & & & & & & & .61 & .66 & .62 & .51 \\
\hline Effort & & & & & & & & & .82 & .80 & .61 \\
\hline Persistence & & & & & & & & & & .79 & .53 \\
\hline Preparation & & & & & & & & & & & .54 \\
\hline
\end{tabular}

Note.: All Correlations are significant at $p<.01 .{ }^{\mathrm{a}}$ Individual Attraction to the Group-Task. ${ }^{\mathrm{b}}$ Individual Attraction to the Group-Social. ${ }^{\mathrm{c}}$ Group Integration Task. ${ }^{\mathrm{d}}$ Group Integration-Social. ${ }^{\mathrm{e}}$ General Task and Communication. ${ }^{\mathrm{f}}$ Team Dynamics Interactions. ${ }^{\mathrm{g}}$ Team Resources and Working Environment. 
Table 3

Model-Data Fit for the Proposed Nomological Network of Team Dynamics in Sport

\begin{tabular}{|c|c|c|c|c|c|c|c|}
\hline Model & $\chi_{S-B}^{2}$ & $D f$ & $\begin{array}{l}\text { Correction } \\
\text { factor }\end{array}$ & CFI & RMSEA & SRMR & WRMR \\
\hline Measurement Model & $55.14^{* *}$ & 30 & 1.21 & .986 & .050 & .026 & .505 \\
\hline \multicolumn{8}{|l|}{ Structural Model } \\
\hline Model 1 & $122.83^{* *}$ & 33 & 1.24 & .950 & .089 & .063 & 1.292 \\
\hline Model 2 & $71.75^{* *}$ & 32 & 1.21 & .978 & .060 & .033 & .611 \\
\hline Model 4 (Final) & $55.79^{* *}$ & 31 & 1.20 & .986 & .048 & .026 & .502 \\
\hline \multicolumn{8}{|c|}{ Measurement Model by Gender } \\
\hline Female Group & $50.40^{* *}$ & 30 & 1.17 & .980 & .062 & .033 & .545 \\
\hline Model Group & 42.66 & 30 & 1.20 & .985 & .051 & .030 & .375 \\
\hline \multicolumn{8}{|c|}{ Two-Sample Measurement Model } \\
\hline Unconstrained Model & $92.94^{* *}$ & 60 & 1.18 & .982 & .057 & .032 & .472 \\
\hline Constrained Model & $95.01^{* *}$ & 66 & 1.20 & .984 & .051 & .036 & .524 \\
\hline \multicolumn{8}{|c|}{ Two-Sample Structural Model } \\
\hline Unconstrained Model & $95.91^{* *}$ & 68 & 1.19 & .985 & .049 & .036 & .518 \\
\hline Constrained Model & $105.26^{* *}$ & 73 & 1.20 & .982 & .051 & .056 & .753 \\
\hline
\end{tabular}

